\title{
Cost-Effectiveness Analysis of Subtraction Scintigraphy in Patients with Acute Lower Gastrointestinal Tract Hemorrhage
}

\author{
Geoffrey M. Currie, CNMT \\ School of Biomedical Sciences, Charles Sturt University, Wagga Wagga, New South Wales, Australia
}

\begin{abstract}
$99 \mathrm{mTc}$-labeled red blood cell scintigraphy is a powerful detection and localization tool that may be confounded by false-positive and false-negative findings. Subtraction scintigraphy has been used in the evaluation of acute lower gastrointestinal tract hemorrhage (LGIH) to reduce the impact of interpretive confounders. The aim of this investigation was to evaluate the cost-effectiveness of the addition of subtraction scintigraphy in the evaluation of patients with acute LGIH. Methods: The clinical phase of this research was a retrospective clinical study with a repeatedmeasures design including randomized control and experimental groups. A total of 49 patient studies were included in the sample. Studies were randomized and interpreted by 4 independent physicians. Decision-tree analysis was used to model direct costs and the potential risks of procedures for 2 diagnostic strategies for patients with acute LGIH: conventional scintigraphy alone and conventional scintigraphy combined with subtraction scintigraphy. The transition probabilities (or branching fraction at each decision node) for scintigraphy were based on the clinical results of this investigation. All other transition probabilities were derived from previously cited data. Results: Combining subtraction techniques with conventional scintigraphy reduced the overall costs of procedures for patients with acute LGIH by $\$ 74$ per patient and reduced deaths by $17.6 \%$ and complications by $15.7 \%$. For conventional scintigraphy alone, $8.8 \%$ of patients presenting for scintigraphic evaluation of acute LGIH would undergo unnecessary angiograms, and $2.8 \%$ would have unnecessary surgery. These figures were reduced to just $5.4 \%$ and $1.8 \%$, respectively, with the addition of subtraction scintigraphy. Conclusion: The use of subtraction scintigraphy as an adjunct to conventional scintigraphy for patients with acute LGIH may provide both cost and outcome benefits.
\end{abstract}

Key Words: bowel hemorrhage; gastrointestinal tract bleeding; subtraction scintigraphy; LGIH

J Nucl Med Technol 2007; 35:140-147

DOI: 10.2967/jnmt.106.037655

\footnotetext{
Received Oct. 26, 2006; revision accepted Jan. 29, 2007.

For correspondence or reprints contact: Geoffrey M. Currie, CNMT, School of Biomedical Sciences, Locked Bag 588, Charles Sturt University, Wagga Wagga 2678, New South Wales, Australia.

E-mail: gcurrie@csu.edu.au

COPYRIGHT @ 2007 by the Society of Nuclear Medicine, Inc.
}

D espite the difficulties confronting the diagnostic evaluation of acute lower gastrointestinal tract hemorrhage (LGIH), accurate localization of the site of bleeding is crucial for treatment and case management $(1,2)$. Although the diagnostic evaluation of acute LGIH has improved significantly over the last $25 \mathrm{y}$, there is currently no gold standard because of the intermittent nature of the bleeding (2-4). ${ }^{99 m}$ Tc-labeled red blood cell (RBC) scintigraphy is a powerful detection and localization tool that may be confounded by false-positive and false-negative findings.

Subtraction scintigraphy has been used in the evaluation of acute LGIH (5). Subtraction scintigraphy should allow the elimination of background activity, providing high target-tobackground images of the extravasated blood. In short, a dataset that theoretically offers the advantages of both conventional ${ }^{99 \mathrm{~m} T c-s u l f u r}$ colloid scintigraphy and ${ }^{99 \mathrm{~m}} \mathrm{Tc}-$ labeled RBC scintigraphy is produced (5).

The aim of this investigation was to compare the costeffectiveness of interpretation of conventional scintigraphy with that of conventional scintigraphy combined with subtraction scintigraphy in the evaluation of patients with acute LGIH.

\section{MATERIALS AND METHODS}

The clinical phase of this research was a retrospective clinical study with a repeated-measures design including randomized control and experimental groups. A total of 49 patient studies were included in the sample. Acquisition parameters for all datasets included a $128 \times 128$ matrix and a continuous dynamic acquisition of $60 \mathrm{~s}$ per frame. Studies were generally performed with RBCs labeled with ${ }^{99 \mathrm{~m}} \mathrm{Tc}$ in vitro by use of a commercially available kit.

The 49 original raw patient datasets were displayed conventionally without subtraction (conventional scintigraphy [CS]), with reference subtraction scintigraphy (RSS), and with alternate sequential subtraction scintigraphy (ASSS). Studies were randomized and interpreted by 4 independent physicians initially as CS data only. The CS, RSS, and ASSS studies were subsequently combined and randomized for reinterpretation. Although studies were randomized within each of the 2 pools of data, CS data were reported before combined CS and subtraction data to remove possible bias. The clinical phase of this research was approved 


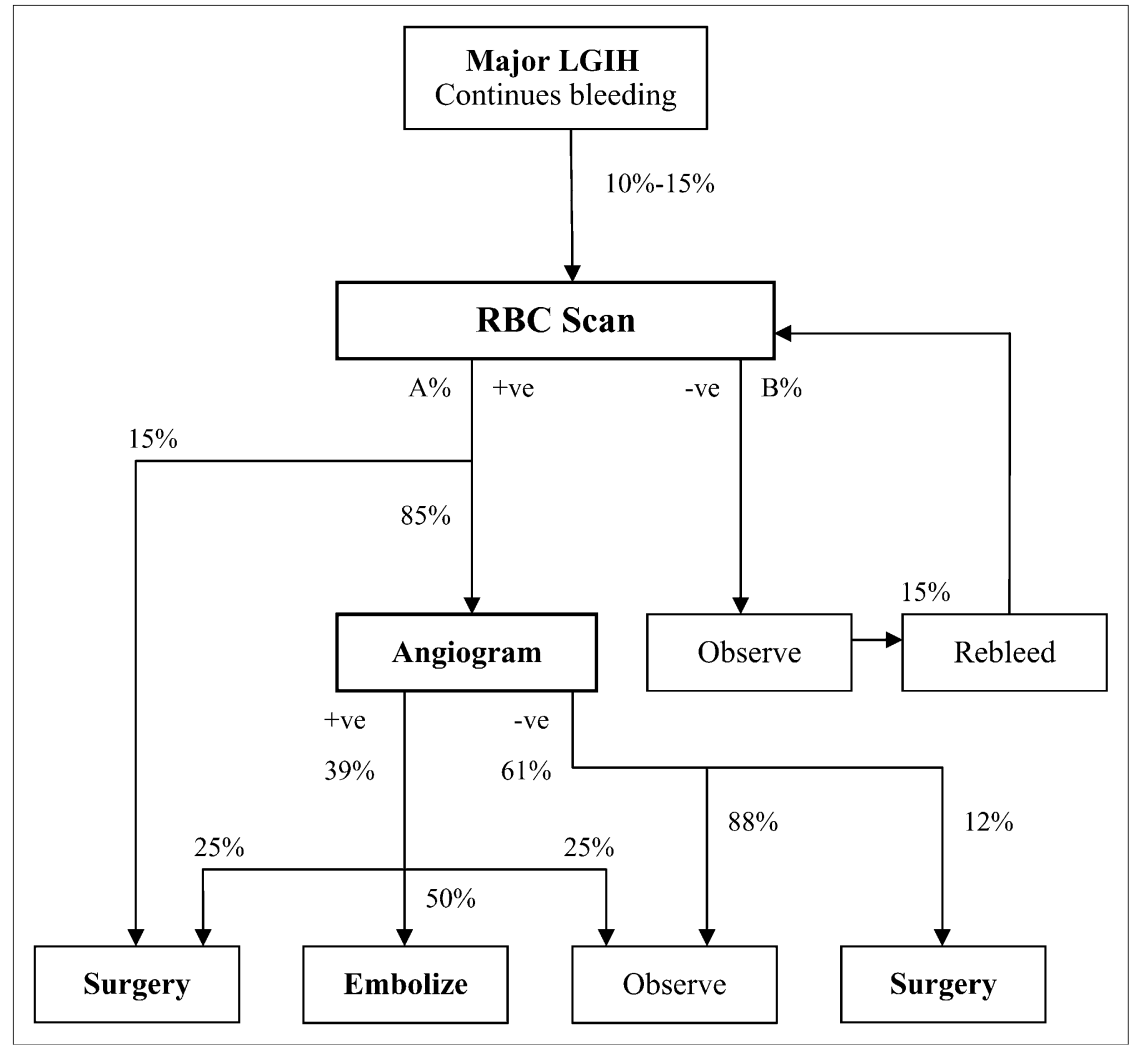

FIGURE 1. Composite diagnostic algorithm for patients with LGIH. A\% and B\% represent alternative diagnostic strategies. $+\mathrm{ve}=$ positive; $-\mathrm{ve}=$ negative .

TABLE 1

Summary of Data Used in Analysis

\begin{tabular}{|c|c|}
\hline Variable & Value (reference) \\
\hline Mean life expectancy & 80.5 y $(7)$ \\
\hline Mortality rate for patients with acute $\mathrm{LGIH}^{\star}$ & $4 \%(3,8)$ \\
\hline Scintigraphy-associated mortality & $0 \%(9)$ \\
\hline Scintigraphy-associated complication rate & $0 \%(9)$ \\
\hline Scintigraphy-associated cost & $\$ 492(6)$ \\
\hline Angiography-associated mortality & $0 \%(3,9,10)$ \\
\hline Angiography-associated complication rate* & $2 \%(3,9,10)$ \\
\hline Angiography-associated cost & $\$ 1,376(6)$ \\
\hline Embolization-associated complication rate ${ }^{*}$ & $0 \%(11)$ \\
\hline Embolization-associated cost & $\$ 144(6)$ \\
\hline Surgery-associated mortality, with localization of bleeding & $13 \%(12,13)$ \\
\hline Surgery-associated mortality, without localization of bleeding & $30 \%(12,13)$ \\
\hline Surgery-associated complication rate, with localization of bleeding & $15 \%(14)$ \\
\hline Surgery-associated complication rate, without localization of bleeding & $83 \%(14)$ \\
\hline Surgery-associated cost & $\$ 1,124(6)$ \\
\hline Surgery as a result of positive scintigraphy & $15 \%(20)$ \\
\hline Angiography as a result of positive scintigraphy & $85 \%(20)$ \\
\hline Positive angiography and positive scintigraphy & $39 \%(20)$ \\
\hline Negative angiography and positive scintigraphy & $61 \%(20)$ \\
\hline Positive angiograms only suggestive of bleeding & $42 \%(20)$ \\
\hline Rate of rebleeding in acute LGIH & $15 \%(15,16)$ \\
\hline Positive angiography leading to surgery & $25 \%(20)$ \\
\hline Positive angiography leading to embolization & $50 \%(20)$ \\
\hline Blind surgery & $12 \%(17)$ \\
\hline
\end{tabular}


TABLE 2

Summary of Clinical Results Used in Analysis

\begin{tabular}{lr}
\hline \multicolumn{1}{c}{ Variable } & Value \\
\hline Mean age of patient cohort & $68.9 \mathrm{y}$ \\
Rate of false-positive findings for CS alone & $10.3 \%$ \\
Rate of false-negative findings for CS alone & $1.1 \%$ \\
Rate of true-positive findings for CS alone & $14.8 \%$ \\
Rate of true-negative findings for CS alone & $73.8 \%$ \\
Rate of false-positive findings for CS and & $4.0 \%$ \\
$\quad$ subtraction & $1.7 \%$ \\
Rate of false-negative findings for CS and & \\
$\quad$ subtraction & $14.4 \%$ \\
Rate of true-positive findings for CS and & $79.9 \%$ \\
$\quad$ subtraction & \\
Rate of true-negative findings for CS and & $8 \%$ \\
$\quad$ subtraction & \\
Negative scintigraphy; rescanned because of & \\
$\quad$ rebleeding & \\
&
\end{tabular}

by the Charles Sturt University Ethics in Human Research Committee.

\section{RSS}

In an acquisition with $n$ consecutive image frames and with each individual image frame given by $F(f)$, where $f$ equals
$(1,2,3, \ldots \ldots \ldots \ldots, n)$, any subtracted image in the sequence is given by the following equation:

$$
S(f)=F(f)-F(1)
$$

For example,

$$
S(10)=F(10)-F(1) .
$$

\section{ASSS}

In an acquisition with $n$ consecutive image frames and with each individual image frame given by $F(f)$, where $f$ equals $(1,2,3, \ldots \ldots \ldots \ldots, n)$, any subtracted image in the sequence is given by the following equation:

$$
S(f)=F(f+1)-F(f-1)
$$

For example,

$$
S(9)=F(10)-F(8)
$$

\section{Decision-Tree Analysis}

Decision-tree analysis was used to model direct costs and the potential risks of procedures for 3 diagnostic strategies for patients with acute LGIH: CS alone, CS combined with subtraction scintigraphy RSS and ASSS, and CS combined with ASSS only. All

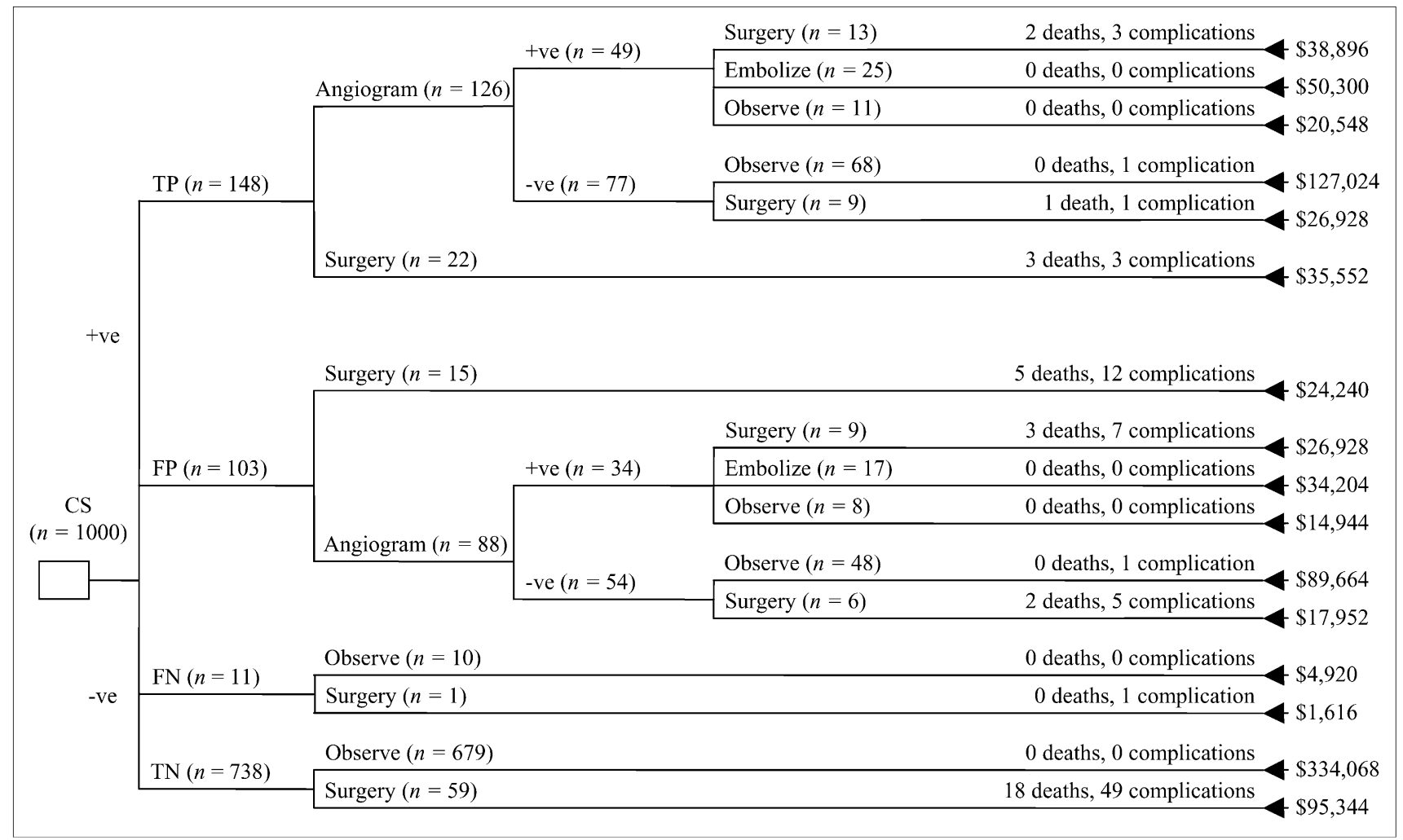

FIGURE 2. Decision-tree analysis for CS alone, with totals of 34 deaths and 83 complications and total cost of $\$ 943,128$. FN $=$ false-negative results; FP = false-positive results; $\mathrm{TN}=$ true-negative results; $\mathrm{TP}=$ true-positive results; + ve $=$ positive; - ve $=$ negative. 


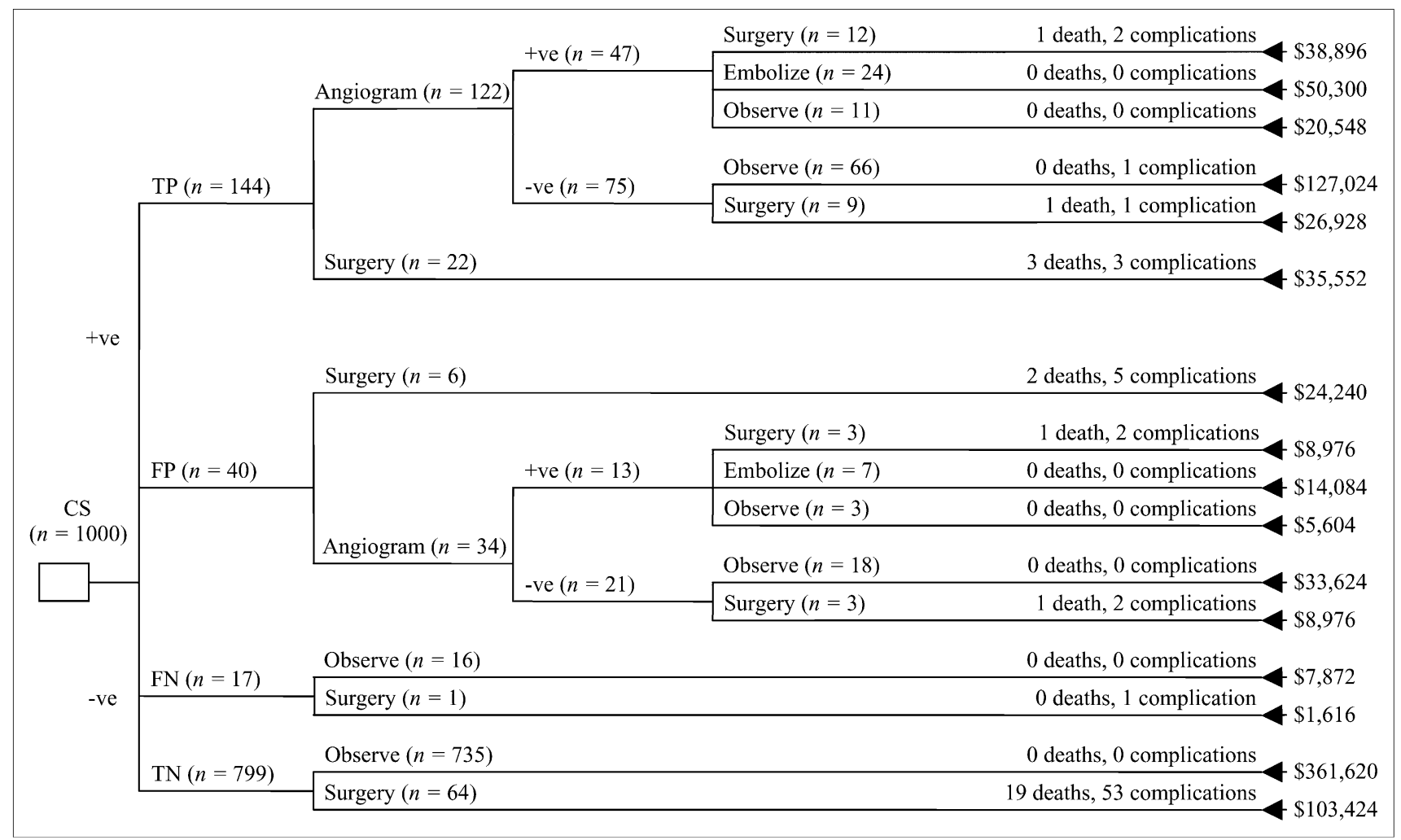

FIGURE 3. Decision-tree analysis for CS, RSS, and ASSS combined, with totals of 28 deaths and 70 complications and total cost of $\$ 869,284$. $\mathrm{FN}=$ false-negative results; $\mathrm{FP}=$ false-positive results; $\mathrm{TN}=$ true-negative results; $\mathrm{TP}=$ true-positive results; + ve $=$ positive; - ve = negative.

diagnostic strategies were based on the diagnostic algorithm depicted in Figure 1.

The decision-tree analysis was based on a hypothetical population of 3,000 patients presenting for scintigraphic evaluation of acute LGIH. Each of 3 diagnostic strategies was used to evaluate 1,000 randomly allocated patients with a homogeneous distribution of variables. The transition probabilities (A\% and B\% in Fig. 1) for scintigraphy were based on the clinical results outlined here. All other transition probabilities (branching fractions at decision nodes) were derived from previously cited data (Table 1). Complication rates and mortality rates were also derived from previously cited data (Table 1). Costs were estimated by use of the Australian Government Medicare Benefits Schedule (6) and are reported in Australian dollars. Table 1 and Table 2 provide an overview of the key information used in the decision-tree analysis and the cost-effectiveness analysis.

Several assumptions were made during this analysis. First, apart from the $15 \%$ of positive scintigraphy studies that led to surgery without angiography, the remaining positive scintigraphy studies all led to angiography. In theory, this scenario is entirely plausible, given the role of scintigraphy in directing patients to undergo angiography, although in practice as many as $45 \%$ of patients with positive scintigraphy studies are simply observed (20). Second, the analysis included a single iteration for cases recirculating in the diagnostic algorithm after an initial negative scintigraphy study. There seemed no tangible benefit of continuing this analysis (negative scintigraphy study-rebleed cycle) ad infinitum. Third, when complication rates and mortality rates were reported in the literature as ranges, with no obvious best value or most recent application, a conservative approach of using the lowest value was taken.

\section{RESULTS}

The mean age of the study population was $68.9 \mathrm{y}(95 \%$ confidence interval: 64.0-73.9 y), with a range of 18.8-92.8 $\mathrm{y}$ and a median of $71.8 \mathrm{y}$. Women represented $61.2 \%$ (30/ 49) of the study population, and men represented $38.8 \%$ (19/49), although no statistically significant variation in the sex distribution was noted $(P=0.11)$. No statistically significant difference between the sexes was noted for mean age $(P=0.50)$. Just 7 patient studies $(14.3 \%)$ were positive for gastrointestinal tract bleeding, as determined by expert panel consensus. The remaining 42 patient studies were not positive for active bleeding at detectable bleeding rates during the data acquisition.

The decision-tree analysis for 1,000 patients evaluated with CS alone (Fig. 2) demonstrated totals of 34 deaths and 83 complications and a total cost of $\$ 943,128$. Eighteen deaths and 49 complications were attributed to blind surgery (i.e., surgery performed without guidance from the results of a diagnostic procedure) being performed on patients with true-negative scintigraphy studies. Further complications were associated with blind surgery being performed on patients with false-negative scintigraphy studies. More 
importantly, 10 deaths and 25 complications were associated with unnecessary procedures being performed on patients with false-positive study results. These findings were associated with an additional cost burden of $\$ 157,256$, which reflects the increase in per-patient cost from $\$ 492$ (truenegative results) to $\$ 2,019$ (false-positive results). The larger cohort with true-positive results had 6 deaths and 8 complications.

The decision-tree analysis for CS, RSS, and ASSS combined (Fig. 3) demonstrated totals of 28 deaths and 70 complications and a total cost of $\$ 869,284$. Nineteen deaths and 53 complications were attributed to blind surgery being performed on patients with true-negative scintigraphy studies. CS, RSS, and ASSS combined reduced deaths in the group with false-positive results from 10 for CS alone to 4 for CS, RSS, and ASSS combined. Furthermore, a corresponding reduction in complications was noted for the group with false-positive results: from 25 to just 9. Although the cost per patient in the false-positive group changed only marginally, reflecting the effects of rounding to whole patient numbers, the total reduction in cost for patients with false-positive results was $\$ 112,428$. The addition of subtraction techniques decreased the overall cost by $\$ 73,844$ ( $\$ 74$ per patient).

The decision-tree analysis for CS and ASSS combined, in which study results were considered positive only when ASSS provided supporting evidence (Fig. 4), demonstrated totals of 26 deaths and 64 complications and a total cost of $\$ 787,344$. These changes reflected the elimination of falsepositive findings and provided a cost saving over CS alone of $\$ 155,784$ ( $\$ 156$ per patient).

Sensitivity analysis is a technique used in economic analysis to assess the robustness of calculations. In costeffectiveness analysis, sensitivity analysis repeats calculations on the basis of worst-case and best-case scenarios to determine whether the conclusions are valid within a broader clinical window. In this cost-effectiveness analysis, sensitivity analysis was used to assess the impact of the variability of proportions reported in Figure 1. The conservative nature of the original analysis virtually provides a worstcase scenario; however, some minor adjustments to proportions in the diagnostic algorithm might negatively affect the diagnostic integrity of scintigraphy (Fig. 5). A best-case scenario can be accomplished by being less conservative with the variables in Table 1 . Table 3 provides a summary of the key variables used in the sensitivity analysis.

The decision-tree analysis for 1,000 patients evaluated with CS alone as a worst-case scenario demonstrated totals of 28 deaths and 70 complications and a total cost of $\$ 757,588$. Six deaths and 16 complications were associated with unnecessary procedures being performed on patients with false-positive study results. The worst-case scenario decision-tree analysis for CS, RSS, and ASSS combined demonstrated totals of 26 deaths and 67 complications and

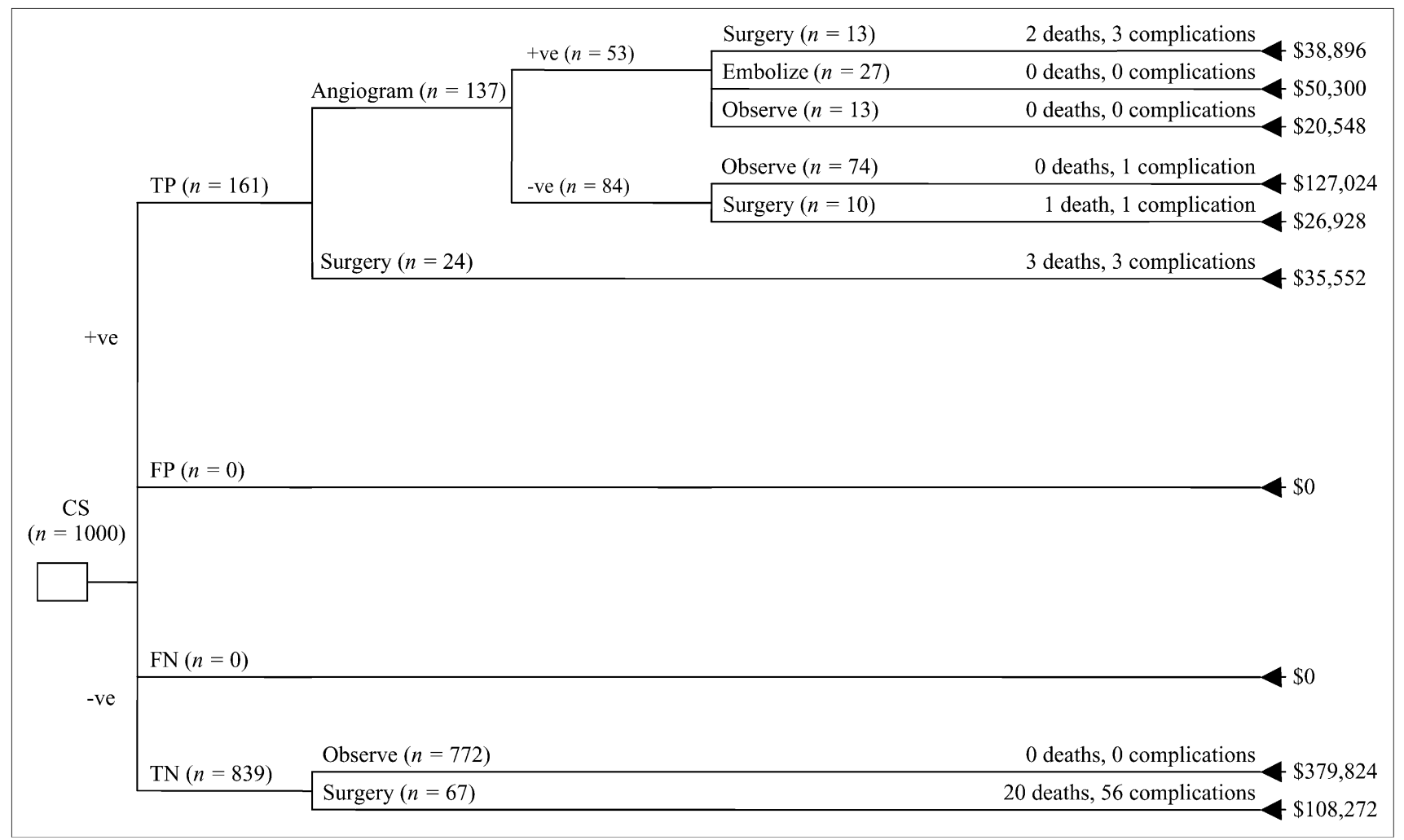

FIGURE 4. Decision-tree analysis for CS and ASSS combined, with totals of 26 deaths and 64 complications and total cost of $\$ 787,344$. FN = false-negative results; FP = false-positive results; $\mathrm{TN}=$ true-negative results; $\mathrm{TP}=$ true-positive results; + ve $=$ positive; - ve = negative. 


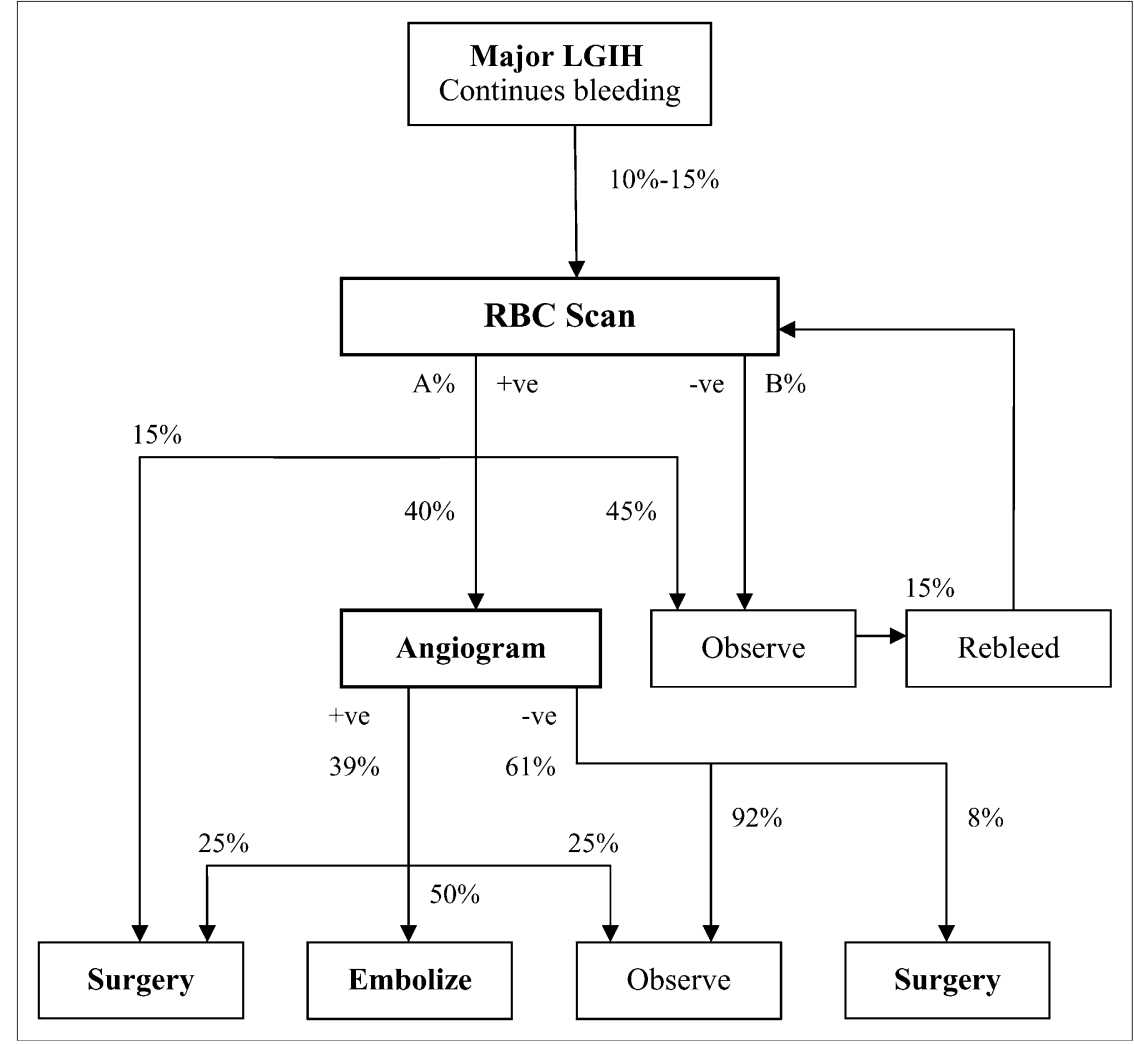

FIGURE 5. Composite diagnostic algorithm for patients with LGIH, with minor adjustments to proportions. $\mathrm{A} \%$ and $\mathrm{B} \%$ represent alternative diagnostic strategies. $+v e=$ positive; $-v e=$ negative. a total cost of $\$ 725,342$. These data corresponded to no changes in deaths or complications in the group with falsepositive results compared with that group evaluated with CS alone. The addition of subtraction techniques reduced the overall cost by $\$ 32,246$ ( $\$ 32$ per patient). The worstcase scenario decision-tree analysis for CS and ASSS combined demonstrated totals of 25 deaths and 63 complications and a total cost of $\$ 692,480$. These changes reflected the elimination of false-positive findings and provided a cost saving over CS alone of $\$ 65,108$ (\$65 per patient).

The decision-tree analysis for 1,000 patients evaluated with CS alone as a best-case scenario demonstrated totals of 38 deaths and 103 complications and a total cost of $\$ 943,128$. Sixteen deaths and 34 complications were associated with unnecessary procedures being performed on patients with false-positive study results. The best-case scenario decisiontree analysis for CS, RSS, and ASSS combined demonstrated

TABLE 3

Summary of Data Used in Sensitivity Analysis

\begin{tabular}{cc}
\hline \multicolumn{1}{c}{ Variable } & Value (reference) \\
\hline Angiogram complication rate & $11 \%(18)$ \\
$\begin{array}{c}\text { Surgery-associated mortality, with } \\
\text { localization of bleeding }\end{array}$ & $10 \%(19)$ \\
$\begin{array}{c}\text { Surgery-associated mortality, } \\
\text { without localization of bleeding }\end{array}$ & $57 \%(19)$ \\
\hline
\end{tabular}

totals of 30 deaths and 83 complications and a total cost of $\$ 869,284$. CS, RSS, and ASSS combined reduced deaths in the group with false-positive results from 16 for $\mathrm{CS}$ alone to 7 for CS, RSS, and ASSS combined. Furthermore, a corresponding reduction in complications was noted for the group with false-positive results: from 34 to just 11 . The addition of subtraction techniques reduced the overall cost by $\$ 73,844$ (\$74 per patient). The best-case scenario decision-tree analysis for CS and ASSS combined demonstrated totals of 24 deaths and 75 complications and a total cost of $\$ 787,344$. These changes reflected the elimination of false-positive findings and provided a cost saving over CS alone of $\$ 155,784$ (\$156 per patient).

\section{DISCUSSION}

The decision trees were burdened by large mortality and complication rates associated with blind surgery being performed for patients with true-negative scintigraphic findings. This finding highlights the role that subtraction scintigraphy might play in improving the negative predictive value of scintigraphy: reducing blind surgery. Nonetheless, the impact of subtraction scintigraphy in reducing the costs and consequences of case management when false-positive results are obtained is highlighted by this analysis.

Combining subtraction techniques with CS not only reduced the overall costs of procedures for patients with acute LGIH but also reduced mortality and morbidity. Combining 
TABLE 4

Results of Sensitivity Analysis of Decision-Tree Data Obtained with Various Subtraction Techniques

\begin{tabular}{|c|c|c|c|c|}
\hline \multirow[b]{2}{*}{ Strategy } & \multirow[b]{2}{*}{ Parameter } & \multicolumn{3}{|c|}{ Reduction compared with CS alone } \\
\hline & & This study & Worst-case scenario & Best-case scenario \\
\hline \multirow[t]{3}{*}{ CS, RSS, and ASSS } & Cost per patient & $\$ 74$ & $\$ 32$ & $\$ 74$ \\
\hline & Complications & 13 & 3 & 20 \\
\hline & Deaths & 6 & 2 & 8 \\
\hline \multirow[t]{3}{*}{ CS and ASSS } & Cost per patient & $\$ 65$ & $\$ 65$ & $\$ 156$ \\
\hline & Complications & 19 & 7 & 28 \\
\hline & Deaths & 8 & 3 & 14 \\
\hline
\end{tabular}

CS, RSS, and ASSS reduced deaths by $6(0.6 \%)$ and complications by $13(1.3 \%)$ while providing a net saving of $\$ 73,844$ over CS alone. Thus, subtraction scintigraphy for patients with acute LGIH provided a saving of $\$ 74$ per patient while saving 6 lives or, on average, 69.6 life years. These data translate to a cost saving of $\$ 12,307$ per life or $\$ 1,061$ per life year.

Combining CS and ASSS reduced deaths by $8(0.8 \%)$ and complications by $19(1.9 \%)$ while providing a net saving of $\$ 155,784$ over CS alone. Thus, ASSS for patients with acute LGIH provided a saving of $\$ 156$ per patient while saving 8 lives or, on average, 92.8 life years. These data translate to a cost saving of $\$ 19,473$ per life or $\$ 1,679$ per life year.

These findings were shown to be robust under the scrutiny of sensitivity analysis, with subtraction scintigraphy offering cost, complication, and mortality reductions even in the worst-case scenario (Table 4). The sensitivity analysis indicated that, with respect to cost reductions at least, the cost-effectiveness of CS, RSS, and ASSS combined was close to that of the best-case scenario, whereas that of CS and ASSS combined was close to that of the worst-case scenario. Thus, the cost benefit of the former may not be fully realized in a clinical model, whereas the latter may afford greater cost savings in a clinical model. It should be noted that "best-case scenario" and "worst-case scenario" refer to the diagnostic integrity of scintigraphy rather than patient outcomes.

In the cohort of patients modeled (clinical-phase data), $8.8 \%$ of the patients presenting for scintigraphic evaluation of acute LGIH would undergo unnecessary angiograms, and $2.8 \%$ would have unnecessary surgery. The former is associated with significant cost and radiation burdens, whereas the latter is associated with significant mortality and morbidity. Combining CS with RSS and ASSS in the clinical phase of this research allowed unnecessary angiograms to be reduced to just $5.4 \%$ of the cohort and unnecessary surgery to be reduced to just $1.8 \%$. Exploiting the distinctive competencies of ASSS in collaboration with CS interpretation and eliminating potential false-positive findings associated with RSS eradicated unnecessary angiograms and surgery altogether. This strategy may introduce a small number of false-negative study results, although this possibility was not revealed in this evaluation. It should be noted that unnecessary surgery and angiography were defined by those procedures performed in cases in which false-positive results were obtained rather than simply those with negative outcomes.

\section{CONCLUSION}

This gastrointestinal tract hemorrhage study with ${ }^{99 \mathrm{~m}} \mathrm{Tc}-$ labeled RBCs demonstrated that false-positive or falsenegative findings may confound interpretation. False-positive findings may lead to a patient unnecessarily undergoing more expensive and higher-risk procedures. Generally, a cost-effectiveness analysis is used to make decisions about the implementation of strategies that add costs while justifying the additional costs with improved outcomes. In this study, subtraction scintigraphy not only improved outcomes but also reduced costs. Subtraction scintigraphy used as an adjunct to conventional scintigraphy for patients with acute LGIH may provide both cost and outcome benefits. Moreover, decision-tree analyses demonstrated the superior cost-effectiveness of CS and ASSS combined over CS alone and over CS, RSS, and ASSS combined. These data reflect the high rate of false-positive findings associated with RSS.

\section{REFERENCES}

1. Maurer AH. Gastrointestinal bleeding. In: Murray IPC, Ell PJ, eds. Nuclear Medicine in Clinical Diagnosis and Treatment. 2nd ed. Vol. 1. London, U.K.: Churchill Livingstone; 1998:67-94.

2. Ziessman HA. The gastrointestinal tract. In: Harbert JC, Eckelman WC, Neumann RD, eds. Nuclear Medicine: Diagnosis and Therapy. New York, NY: Thieme Medical Publishers Inc.; 1996:585-670.

3. Peter DJ, Dougherty JM. Evaluation of the patient with gastrointestinal bleeding: an evidence based approach. Emerg Med Clin North Am. 1999;17:239-261.

4. Palmer EL, Scott JA, Strauss HW. Practical Nuclear Medicine. Philadelphia, PA: WB Saunders Co.; 1992.

5. Currie GM, Towers PA, Wheat JM. Improved detection and localization of lower gastrointestinal tract hemorrhage by subtraction scintigraphy: phantom analysis. J Nucl Med Technol. 2006;34:160-168.

6. Australian Government. Medicare Australia. Medicare Benefits Schedule. Available at: http://www.medicareaustralia.gov.au/. Accessed July 15, 2007.

7. Australian Bureau of Statistics. Year Book Australia 2006. Available at: http:// www.abs.gov.au. Accessed July 15, 2007.

8. Longstreth GF. Epidemiology and outcome of patients hospitalized with acute lower gastrointestinal hemorrhage: a population based study. Am J Gastroenterol. 1997;92:419-424. 
9. O'Neill BB, Gosnell JE, Lull RJ, et al. Cinematic nuclear scintigraphy reliably directs surgical intervention for patients with gastrointestinal bleeding. Arch Surg. 2000;135:1076-1081.

10. Balduf LM, Langsfeld M, Marek JM, Tullis MJ, Kasirajan K, Matteson B. Complication rates of diagnostic angiography performed by vascular surgeons. Vasc Endovascular Surg. 2002;36:439-445.

11. Hastings GS. Angiographic localization and transcatheter treatment of gastrointestinal bleeding. RadioGraphics. 2000;20:1160-1168.

12. Punamiya SJ, Someshwar VR. Angiography in the diagnosis and treatment of lower gastrointestinal bleeding. Bombay Hosp J. 2000;42(special issue). Available at: http://www.bhj.org/journal/2000_4202_apr00/sp_289.htm. Accessed July 15, 2007.

13. Wojtowycz M. Handbook of Interventional Radiology and Angiography. 2nd ed. St. Louis, MO: Mosby; 1995.

14. Parkes BM, Obeid FN, Sorensen VJ, Horst HM, Fath JJ. The management of massive lower gastrointestinal bleeding. Am Surg. 1993;59:676-678.
15. Al Qahtani AR, Satin R, Stern J, Gordon PH. Investigative modalities for massive lower gastrointestinal bleeding. World J Surg. 2002;26: 620-625.

16. Zuckerman GR, Prakash C. Acute lower intestinal bleeding, part I: clinical presentation and diagnosis. Gastrointest Endosc. 1998;48:606-617.

17. Enns R. Acute lower gastrointestinal bleeding: part 1. Can J Gastroenterol. 2001;15:509-516.

18. Cohn SM, Moller BA, Zieg PM, Milner KA, Angood PB. Angiography for preoperative evaluation in patients with lower gastrointestinal bleeding: are the benefits worth the risks? Arch Surg. 1998;133:50-55.

19. Green BT, Rockey DC. Lower gastrointestinal bleeding: management. Gastroenterol Clin North Am. 2005;34:665-678.

20. Olds GD, Cooper GS, Chak A, Sivak MV, Chitale AA, Wong RC. The yield of bleeding scans in acute lower gastrointestinal hemorrhage. J Clin Gastroenterol. 2005;39:273-277.

\section{Erratum}

In the article "An Introduction to $\mathrm{Na}^{18} \mathrm{~F}$ Bone Scintigraphy: Basic Principles, Advanced Imaging Concepts, and Case Examples," by Bridges et al. (J Nucl Med Technol. 2007;35:64-76), the legends of Figure 1 (which is also the cover image) and Figure $6 \mathrm{E}$ mistakenly describe the image as having been obtained with ${ }^{18} \mathrm{~F}-\mathrm{FDG}$ instead of with the correct tracer, ${ }^{18} \mathrm{~F}$. We regret the error. 